\title{
Alexa Skills como herramientas de apoyo en una clase de Inglés para el desarrollo de las habilidades de Speaking y Listening
}

\section{Alexa Skills as support tools in an English class for developing Speaking and Listening skills}

CORDOVA-OSORIO, Luis Alberto†, ORTEGA-GINES, Héctor Bernardo, ESCOBEDO-GARCIA, Juan Diego y GALICIA-GARCIA, Christian

Universidad Tecnológica de Tehuacán. Prolongación de la 1 sur No. 1101 San Pablo Tepetzingo, 75859 Tehuacán, Pue.

ID $1^{\text {er }}$ Autor: Luis Alberto, Cordova-Osorio / ORC ID: 0000-0003-1330-6431, Researcher ID Thomson: X-8415-2018, CVU CONACYT ID: 954129

ID $1^{\mathrm{er}}$ Coautor: Héctor Bernardo, Ortega-Gines / ORCID: 0000-0003-0877-2227, CVU CONACYT ID: 687065

ID $2^{\text {do }}$ Coautor: Juan Diego, Escobedo-Garcia / ORC ID: 0000-0008-6679-0418, Researcher ID Thomson: X-8555-2018, CVU CONACYT ID: 954160

ID $3^{\text {er }}$ Coautor: Christian, Galicia-Garcia / ORC ID: 0000-0001-7796-4295, Researcher ID Thomson: X-6362-2018, CVU CONACYT ID: 618470

DOI: $10.35429 / J B E .2019 .10 .3 .15 .20$

Recibido 10 de Octubre, 2019; Aceptado 18 de Diciembre, 2019

\section{Resumen}

El proyecto tiene como objetivo principal utilizar Alexa skills para que mediante comandos de voz (intents) se puedan generar experiencias más personalizadas en el aula de clases durante la enseñanza del idioma Inglés. La skills programadas están orientadas a desarrollar dos de las cuatro habilidades principales que debe adquirir un estudiante al aprender un idioma como segunda lengua, "Speaking" y "Listening". Como primer paso de la metodología de desarrollo se seleccionaron los temas de mayor dificultad de entender para cada estudiante, posteriormente con ayuda de los docentes de Inglés se generaron diferentes modelos de conversaciones. Para el tercer paso se programaron las "skills" con las conversaciones generadas. Finalmente se probaron en el aula de clases, con estudiantes de nivel intermedio. Este proyecto ha probado a hacer mas dinamicas las clases del idioma Inglés, así mismo ha permitido que los alumnos desarrollen sus habilidades de "Speaking" y "Listening" de una manera más natural.

\begin{abstract}
The project has as main objective to use Alexa skills in order to generate more personalize experiences (intents) within a classroom in the English Language teaching creating voice commands. The programmed skills are meant to develop two out of four main abilities a student must acquire when learning English as a second language, Speaking and Listening. The first step in the development methodology was to select the topics the students find more difficult to learn, after that with the help of English teachers different conversation models were generated. In the third step the skills were programmed based on the conversations previously generated. Finally the skills were tested in a classroom by intermediate level students. This project has proved to make English class more interactive, it has also allowed students to develop speaking and listening skills in a more natural way.
\end{abstract}

Skills, intents, listening

Citación: CORDOVA-OSORIO, Luis Alberto, ORTEGA-GINES, Héctor Bernardo, ESCOBEDO-GARCIA, Juan Diego y GALICIA-GARCIA, Christian. Alexa Skills como herramientas de apoyo en una clase de Inglés para el desarrollo de las habilidades de Speaking y Listening. Revista de Educación Básica. 2019. 3-10: 15-20.

$\dagger$ Investigador contribuyendo como primer autor. 


\section{Introducción}

Actualmente estamos inmersos en un mundo globalizado, donde el lenguaje ha adquirido gran relevancia como medio de desarrollo intelectual, la supremacía actual del inglés como lengua internacional, ha hecho surgir la segregación lingüística (Gutiérrez \& Landeros, 2010), es por ello que el aprender el idioma inglés es de suma importancia. Existen cuatro habilidades que desarrollar en el inglés: escuchar, leer, hablar y escribir, el presente proyecto se centra en el desarrollo de dos habilidades principales, citadas según Harmer, las señala de la siguiente manera; Listening: La escucha debe ser de manera extensa e intensa, ya que de esa manera el estudiantado puede mejorar sus habilidades y obtener valiosa información del lenguaje a través de una combinación de materiales y procedimientos de escucha extensiva e intensiva.

Speaking: capacidad de hablar con fluidez presupone no sólo el conocimiento de las características del lenguaje, sino también la capacidad para procesar información y lenguaje "en el acto". (Harmer, 2011). Las formas tradicionales en la que estas habilidades se desarrollan en los estudiantes es variada, el presente proyecto se sugiere una no convencional, el uso de una tecnología llamada Alexa Skills de Amazon, esto es una herramienta de comandos de voz que permite una interacción oral en dos vias. El uso de esta herramienta permite que los estudiantes desarrollen sus habilidades de Speaking y Listening de forma mas natural y en escenarios mas reales.

\section{Objetivos}

\section{Objetivo General}

- Desarrollar un conjunto de skills utilizando Alexa de Amazon para el desarrollo de las habilidades "Speaking" y "Listening" en el idioma inglés.

\section{Objetivos Específicos}

- Generar diferentes conversaciones modelo con ayuda de los docentes de la asignatura de inglés.

- Adecuar las conversaciones a la sintaxis de una skill con ayuda de la documentación oficial.

ECORFAN $^{\circledR}$ Todos los derechos reservados
- Programar el catálogo de "intents" para poder interactuar con Alexa.

\section{Marco Teórico}

Alexa.

Alexa es un servicio de voz basado en la nube de Amazon, disponible para mas de 100 millones de dispositivos de Amazon y dispositivos de otros fabricantes. Con Alexa, se pueden construir experiencias de voz de forma natural que ofrecen a los clientes un modo mas intuitivo de interactuar con la tecnología que usan todos los días. (Amazon, https://developer.amazon.com/alexa, 20102019).

Skills.

Alexa provee un conjunto de habilidades conocidas como "skills". Por ejemplo dentro de sus habilidades se incluye la posibilidad de reproducir musica de diferentes proveedores, responder ppreguntas, proveer pronosticos del clima, y consultar Wikipedia. (Amazon, developer.amazon.com, 2010-2019). Alexa Skills Kit.

\section{Alexa Skills Kit.}

Alexa Skills Kit te permite enseñar a Alexa nuevas habilidades. Los usuarios pueden acceder a las nuevas habilidades haciendo a Alexa preguntas o haciendo peticiones. Se pueden construir skills que le den a los usuarios diferentes tipos de habilidades. Por ejemplo, una habilidad podría controlar las luces de una habitación o inicar un juego. (Amazon, https://developer.amazon.com/alexa, 20102019)

\section{Metodología a desarrollar}

Alexa es un software de reconocimiento de voz que funciona principalmente en dispositivos "echo" de Amazon. Alexa se activa al "escuchar" la palabra "alexa", conocida como "wake word", dando un lapso de 8 segundos para recibir la instrucción. Las intrucciones estan dadas por una accion (verbo o "launch") que se quiere efectuar (ver figura 1) y el nombre de la skill o "invication name", dando como resultado una accion oral por parte de Alexa, previamente configurada. 
"Alexa, say hi
to my students"

Hello everyone my name is alexa, how can I help you?

Figura 1 Interaccion con Alexa. El usuario activa Alexa, posteriormente recibe una respuesta

\section{Inicio}

En primer lugar para el desarrollo del proyecto se hizo la selección de los contenidos temáticos, basándose en las hojas de asignatura de inglés y en los resultados de los diferentes exámenes de diagnóstico para determinar que temas son en los que los alumnos y alumnas tienen más problemas y poder abordarlos de una forma más interactiva con Alexa Skills. Se optó por empezar a crear las "skills" para el nivel básico, debido a que los de nuevo ingreso vienen con algunas carencias y poder nivelarlos, además se busca que los alumnos de nuevo ingreso se mantengan motivados en el aprendizaje del idioma utilizando una herramienta nueva. Posteriormente, se analizaron los temas para determinar que tipo de "skills" generar, es decir, que escenarios se podían plantear a un alumno o alumna para iniciar una conversación que se pueda mantener de forma fluida.

\section{Planeación}

En esta etapa del proyecto se asignaron actividades a los diferentes participantes, así mismo se pidió la ayuda a los docentes de la asignatura de inglés. Se generó una tabla con las diferentes unidades temáticas y los temas (ver tabla 1) para posteriormente realizar las conversaciones.

\begin{tabular}{|l|l|l|}
$\begin{array}{l}\text { Unidad } \\
\text { temática }\end{array}$ & \multicolumn{2}{|l|}{$\begin{array}{l}\text { Tiempo } \\
\text { verbal }\end{array}$} \\
\hline Presentación & $\begin{array}{l}\text { Saludos, presentación } \\
\text { personal }\end{array}$ & $\begin{array}{l}\text { Presente: To } \\
\text { Be }\end{array}$ \\
\hline Actividades & $\begin{array}{l}\text { Actividades } \\
\text { cotidianas, } \\
\text { ubicaciones }\end{array}$ & $\begin{array}{l}\text { Presente } \\
\text { simple }\end{array}$ \\
\hline $\begin{array}{l}\text { El presente } \\
\text { continuo }\end{array}$ & $\begin{array}{l}\text { Actividades } \\
\text { progreso }\end{array}$ & $\begin{array}{l}\text { Presente:To } \\
\text { Be y -ing }\end{array}$ \\
\hline
\end{tabular}

Tabla 1 Temas a desarrollar. Listado de temas y tiempos verbales para generar conversaciones

\section{Ejecución}

Para esta etapa del proyecto se revisaron las conversaciones generadas por los docentes, para poderlas convertir a Alexa "skills", es decir establer el formato correcto para su uso en el dispositivo, bajo el formato que se muetra en la figura 2.

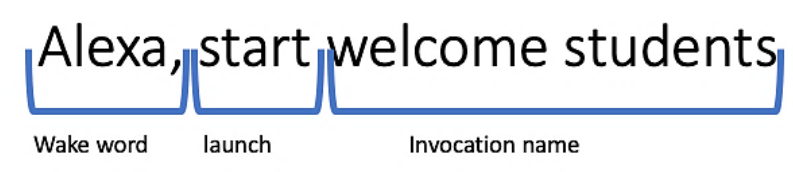

Figura 2 Partes de un skill. Cada skill debe tener tres elementos base para poder ser ejecutado

Cabe mencionar que en la sección de "launch word", pueden ser establecidas diferentes palabras, como los son: "open", "run", "do", "launch", etc. Esta palabra es la que activa el uso de una skill especifica. Para el desarrollo se creó una cuenta en la plataforma de desarrollo de Alexa Skills (ver figura 3)

amazon alexa

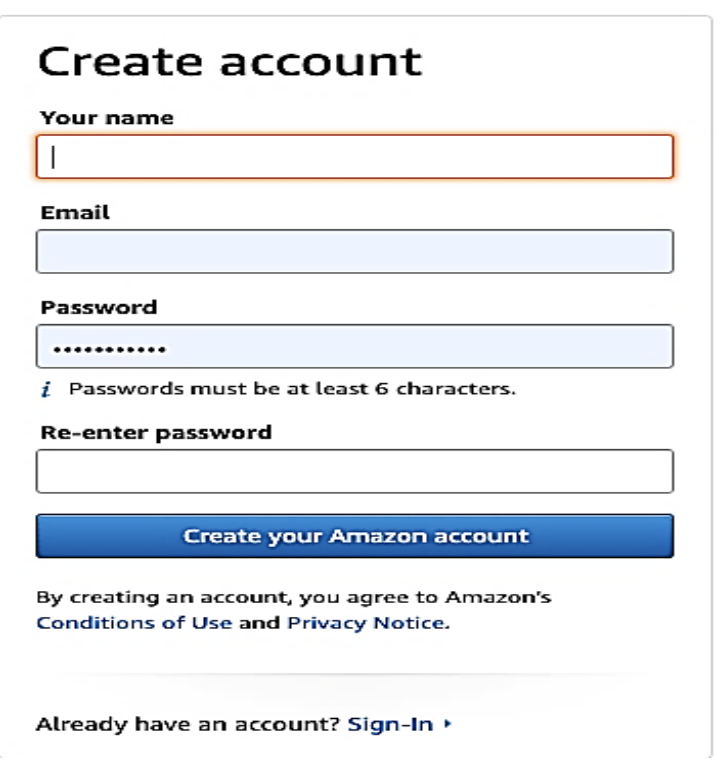

Figura 3 Registro de nueva cuenta. Se deben ingresar los datos para poder proceder a la consola de desarrollo

Una vez creada y activada la cuenta, en la consola se creó una nueva "skill", asignando un nombre para su identificación. A continuación se seleccionó el idioma inglés para que la pronunciación sea la correcta debido a la forma en que se manipula por los alumnos y alumnas (ver figura 4 ). 
Create a new skill

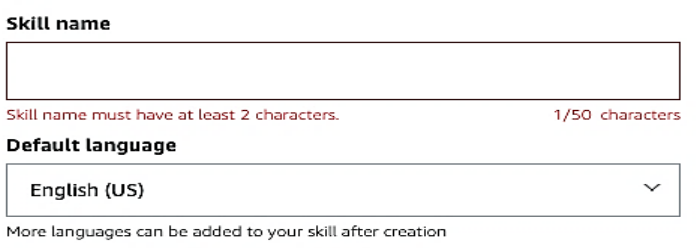

Figura 4 Asignación de nombre e idioma. El nombre debe tener al menos 150 letras y el idioma para este caso es inglés

Posteriormente se seleccionó la categoria de "custom", ya que es una skill perzonalizada e iniciada desde cero. Finalmente se activó la opción de "Alexa hosted", pues los servicios que consume el dispositivo son funciones que ofrece Amazon Web Services. Para la creación de una nueva skills en la consola de desarrollo se deben satisfacer diferentes etapas (ver figura 5). Es importente hacer mención que por cada estapa realizada se deben guardar cambios.

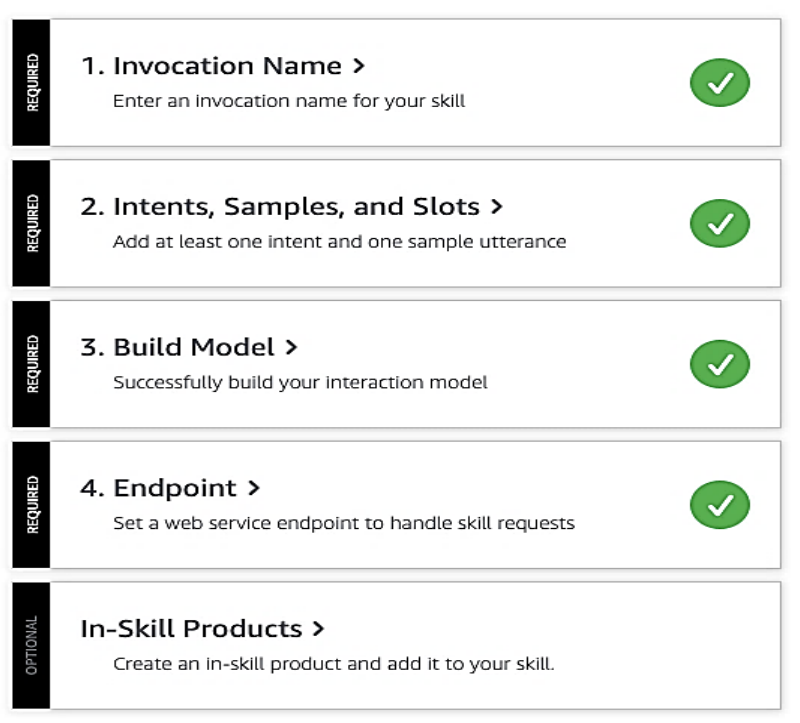

Figura 5 Etapas de desarrollo de una skill. Cada etapa permite debe ser completada correctamente

\section{Etapa "Invocation Name"}

En esta etapa se declara el nombre bajo el cual la "skill" es invocada. (ver figura 6)

Skill Invocation Name (?)

welcome students

Figura 6 Invocation Name. Bajo este nombre Alexa sabe que realizar

\section{Etapa "Intents"}

Un "Intent" es la declaración de una o varias frases, es decir, un catálogo de "utterances" u oraciones que Alexa pueda reconocer por parte de un alumno o alumna como posibles respuestas. Como se observa en la figura 7 , se declaró un intent con tres "utterances".

\section{Intents / welcomeStudents}

Sample Utterances (3) (2)

What might a user say to invoke this intent?

how are you doing

how is it going

how are you

Figura 7 Declaración de intent con utterances. El intent llamado welcomeStudents tiene tres utterancres

\section{Etapa "Build Model"}

Dentro de esta etapa simplemente se realiza una compliación que agrega los intents al contexto de la "skill". La compilación se hace con ayuda de la consola de desarrollo.

\section{Etapa "Handler"}

Una vez concluida la etapa anterior, se crean los "Handlers", debido a que en la configuración inicial de este proyecto se seleccionó "hosted skill", de forma automatica la plataforma de desarrollo crea un "handler". El desarrollo se puede realizar con diferentes lenguajes de programación. Para este proyecto se utilizó Python. Dentro de este archivo se maneja una funcion principal o "handler" que inicia la interacción con el estudiante(ver figura 8), las opciones de lo que se espera "escuchar" deben estar relacionadas al "intent" declarado previamente.

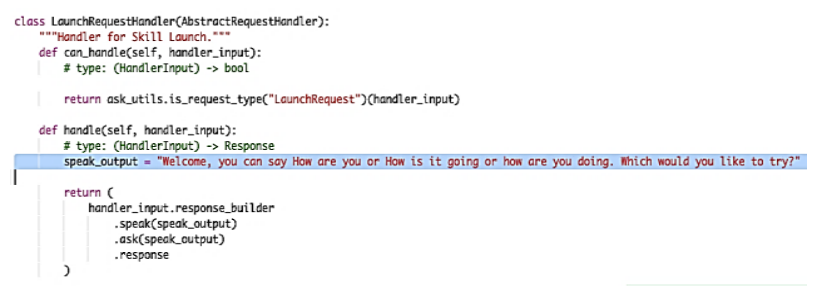

Figura 8 Declaración del handler. Se establece el texto que Alexa repite 
Dentro de esta sección se encuentra tambien una función donde se puede configurar la respuesta que alexa vocaliza, en caso de "escuchar" alguno de los "utterances" declarados en el "intent" como se puede ver en la figura 8 .

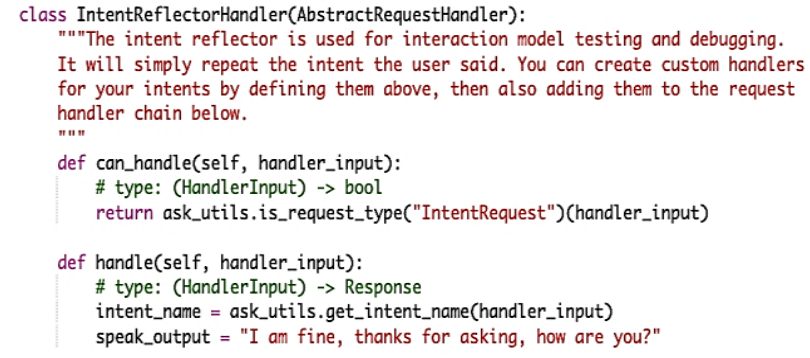

Figura 9 Declaración de respuesta. Si Alexa recibe un patron conocido, responde de acuerdo con lo configurado en esta función

En caso de que Alexa no reciba ningun estimulo o "uterance" previamente agregado entonces se activa la funcion que se observa en la figura 10. Un mensaje que haga saber al estudiante que no se comprende lo dicho.

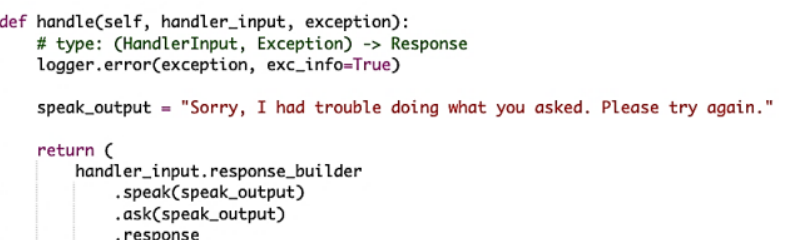

Figura 10 Función de excepciones. Configuración de las excepciones no agregadas como "utterances" o si hubo alguna interferencia al recibir la orden

Existe también una función que permite la cancelación de la skills por parte del estudiante (ver figura 11)

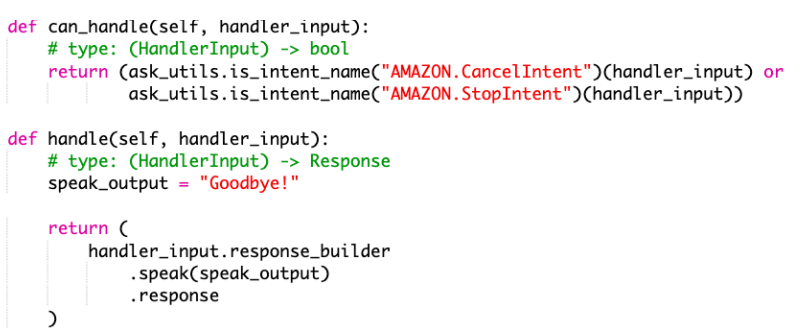

Figura 11 Función para cancelar una skill. Permite la finalización de la skill, usando un Cancel o un Stop

\section{Etapa "Test"}

Para la verificación de las skills, se realizaron pruebas con ayuda de la consola de desarrollo, ya que para la interacción se puede hacer uso del microfono de una PC o una laptop (ver figura 9).
Como parte de la configuración, para activar a Alexa, se hace uso de la "wake word" y el "launch" seguido del "invocation name". Esto activa el "handler", que le da al estudiante información para poder continuar con la conversación o "skill".

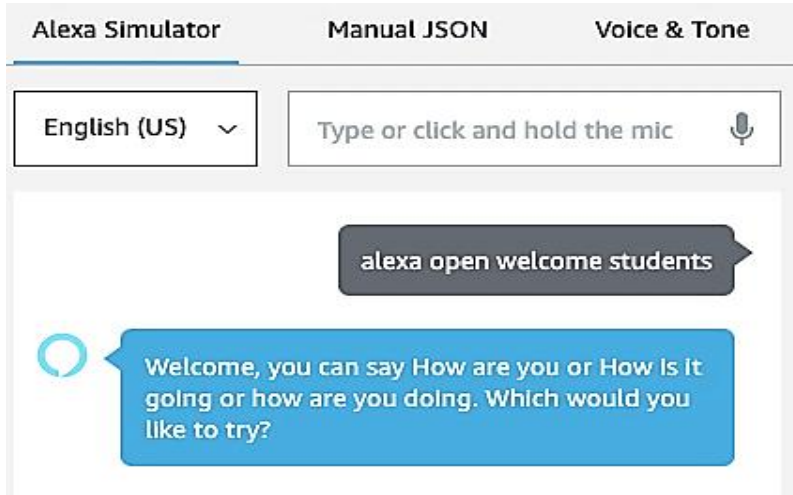

Figura 12 Prueba de la "Skill". El estudiante dice "Alexa open welcome students", para iniciar la interacción

Una vez escuchada por el estudiante la participación de Alexa, el estudiante debe usar una de la opciones dadas para poder continuar con la interacción (ver figura 13), de lo contrario Alexa hace saber que no puede continuar ya que no pudo entender lo dicho o simplemente no le fue configurada la opción (ver figura 14).

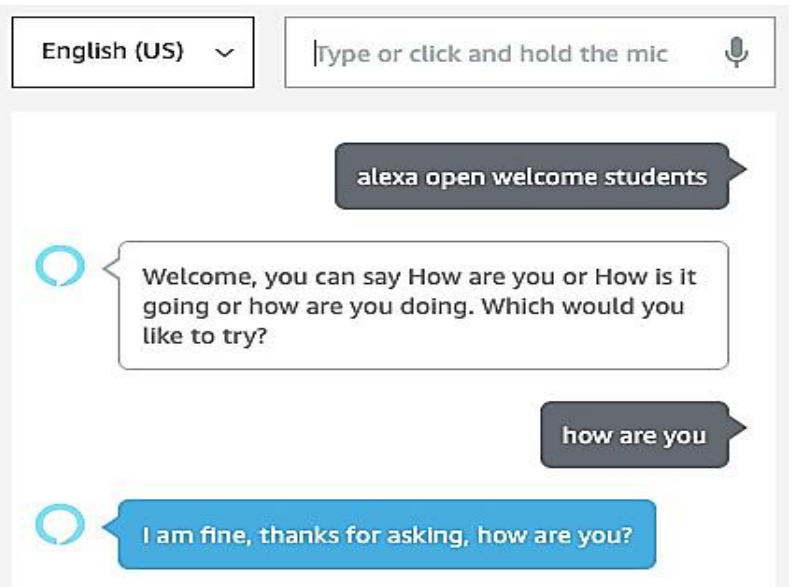

Figura 13 Segunda interacción con Alexa. El usuario contesta con una "utterance" conocida para poder continuar

\section{which do you prefe}

\section{Sorry, I'm not sure.}

Figura 14 Utterance no encontrado. Cuando Alexa no tiene guardado un utterance, la interacción no se detiene, sino que hace saber al estudiante información 
Si el estudiante lo desea también puede terminar la interacción usando el comando "Cancel" o "Stop", como se observa en las figuras 15 y 16.

\section{cance}

Goodbyel

Figura 15 Finalización de skil. La skills es finalizada mediante el comando "cancel"

\section{stop}

Goodbye

Figura 16 Finalización de skill. La skills es finalizada mediante el comando "stop"

\section{Resultados}

Se obtuvó un grupo de skills a partir de un conjunto de conversaciones generadas por diferentes docentes de la asignatura del idioma inglés. Al tener las conversaciones listas se tuvo como primer reto el adecuar dichas conversaciones bajo el patron necesario según el contexto de una skill. Una vez superado ese reto se logró que las skills se pudieran terminar satisfacctoriamente para continuar con las pruebas. Para probar la funcionalidad se trabajó con un grupo para ver que mejoras se pudieran realizar, así como verficar posibles errores durante una fase de producción. El principal problema que se solucionó, fueron errores de redacción. Se debe tomar en cuenta que la mayor parte del tiempo Alexa utiliza machine learning, que significa que usa probalidades de la mayor similitud para así predecir lo que el usuario va a decir, lo cual permite un dialogo mas fluido pues algunas veces los estudiantes no realizan correctamente una pronunciación.

\section{Conclusiones}

Se puede concluir que el uso de la herramienta Alexa Skills dentro del aula de clases, es muy favorable, para ayudar a un estudiante en el desarrollo de las habilidades "Speaking" y "Listening" del idioma inglés. La integración de Alexa skills es sencilla pues el dispositivo esta siempre disponible para ser activado durante la clase de forma natural.
Otro factor que permite que Alexa sea muy buena opción es que no es un simple software de reconocimiento de voz, sino que es una inteligencia artifical muy potente. Finalmente los alumnos y alumnas son los principales benficiados con este proyecto pues ahora pueden practicar y desarrollar sus habilidades en el idioma de una manera mas fluida.

\section{Referencias}

Amazon. (Julio de 2010-2019). developer.amazon.com. Obtenido de https://developer.amazon.com/docs/askoverviews/build-skills-with-the-alexa-skillskit.html

Amazon. (2010-2019). https://developer.amazon.com/alexa. Recuperado el Julio de 2019, de developer.amazon.com:

https://developer.amazon.com/alexa

Amazon. (s.f.). developer.amazon. Recuperado el 4 de 09 de 2019, de https://developer.amazon.com:

https://developer.amazon.com/docs/askoverviews/build-skills-with-the-alexa-skillskit.html

Gutiérrez, R. M., \& Landeros, F. I. (2010). Importancia del lenguaje en el contexto de la aldea global (Vol. 15). Chillán, Chile: Horizontes Educacionales.

Harmer, J. (2011). How To Teach English. Harlow, England: Pearson Longman. 